\title{
The effect of friction on wide shear bands
}

\section{S. LUDING}

Particle Technology, Nanostructured Materials, DelftChemTech, TU Delft, Julianalaan 136, 2628 BL Delft, Netherlands

new address: Multi Scale Mechanics, CTW, UTwente, P.O.Box 217, 7500 AE Enschede, Netherlands

e-mail: s.luding@utwente.nl

\section{Abstract}

Frictional and frictionless granular materials in a split-bottom ring shear cell geometry show wide shear bands under slow, quasi-static deformation. Here, the differences between frictional and frictionless materials are elaborated using discrete element simulations (DEM). Several continuum fields like the density, the velocity field, the deformation gradient and the stress are used here for comparison.

Interestingly, the shear stress intensity, i.e., the shear stress divided by the pressure, is approximately constant throughout the wide shear band, as long as the strain rate is large enough - indicating a Mohr-Coulomb type yield stress fluid. The "viscosity", i.e., the shear stress divided by the strain rate, is increasing with the pressure - which is proportional to the contact number density. Furthermore, the viscosity is inversely proportional to the non-dimensional strain-rate - indicating shear softening behavior inside the wide shear bands.

Keywords: Quasi-static Shear Flow rheology, shear thinning, friction, shear band, discrete element simulation (DEM), viscosity, yield-stress

\section{Introduction}

Granular materials consist of many independent particles with peculiar flow behavior. Segregation, clustering, shear-band formation and arching are some examples. Particle properties and interaction laws can be inserted into a discrete element model (DEM), which then follows the evolution of Newtons equations of motion for the dissipative, frictional many-particle system under observation (Herrmann, Hovi, and Luding 1998; Kishino 2001; Luding, Lätzel, and Herrmann 2001; Luding 2004c).

One goal can be to obtain continuum constitutive relations as needed for 
industrial design. For this, methods and tools for a so-called micro-macro transition are available (Vermeer, Diebels, Ehlers, Herrmann, Luding, and Ramm 2001; Lätzel, Luding, and Herrmann 2000; Luding 2004a; Luding 2005b; Luding 2005a). "Microscopic" simulations of homogeneous small samples, i.e., socalled representative volume elements (RVE) can be used to derive macroscopic constitutive relations needed to describe the material within the framework of a continuum theory (Vermeer, Diebels, Ehlers, Herrmann, Luding, and Ramm 2001). An alternative is to simulate an inhomogeneous geometry where static and dynamic, flowing zones co-exist and, respectively, high density co-exists with dilated zones. From adequate local averaging over equivalent volumes - inside which all particles behave similarly - one can obtain from a single simulation already constitutive relations in a certain parameter range, as was done systematically in two-dimensional Couette ring shear cells (Lätzel, Luding, and Herrmann 2000; Lätzel, Luding, and Herrmann 2001; Lätzel, Luding, Herrmann, Howell, and Behringer 2003) and bi-axial geometries (Luding 2005b; Luding 2005a). Note that both time- and space- averaging are required to obtain a reasonable statistics.

In this study, the micro-macro averaging is applied to a three-dimensional split-bottom shear cell as recently examined experimentally (Fenistein and van Hecke 2003; Fenistein, van de Meent, and van Hecke 2004) and theoretically (Unger, Török, Kertész, and Wolf 2004; Depken, van Saarloos, and van Hecke 2006; Cheng, Lechman, Fernandez-Barbero, Grest, Jaeger, Karczmar, Möbius, and Nagel 2006; Török, Unger, Kertesz, and Wolf 2007). Some concepts from the GDR MiDi report (GDR MiDi 2004) on dense granular flows are used and discussed for a frictionless and a frictional granular material.

The special property of a split-bottom ring shear cell is the fact that the shear band is initiated at the bottom slit and its velocity field is well approximated by an error-function (Fenistein, van de Meent, and van Hecke 2004; Luding 2004c; Luding 2006b) with a width considerably increasing from bottom to top (free surface). Simulations with frictionless particles were compared to experimental data and quantitative agreement was found, with systematic deviations of the order of 20 per-cent. In this study, the frictionless data are compared to results from a simulation with friction. We discuss the quantitative agreement/disagreement, give possible reasons, and outline further research perspectives. 


\section{The Soft Particle Molecular Dynamics Method}

The behavior of granular media is simulated with the discrete element method (DEM) or molecular dynamics (MD) (Allen and Tildesley 1987; Rapaport 1995; Herrmann, Hovi, and Luding 1998; Vermeer, Diebels, Ehlers, Herrmann, Luding, and Ramm 2001; Lätzel, Luding, Herrmann, Howell, and Behringer 2003; Luding 2004b; Luding 2004c; Luding 2006a; Ries, Wolf, and Unger 2007; Luding 2008). Note that both methods are identical in spirit, however, different groups of researchers use these (and also many other) names. The essential ingredient is the force-displacement relation that governs the interaction between pairs of particles. Particle positions, velocities and interaction forces are then sufficient to integrate Newtons equations of motion. Conceptually, the MD or DEM method has to be separated from the hard sphere event-driven (ED) molecular dynamics, and also from the so-called Contact Dynamics (CD), which are not discussed here in detail. Note that in MD or DEM the particles are soft, deformable, while they are rigid in the other two methods.

Since the realistic modeling of the deformations of the particles is much too complicated, we relate the normal interaction force to the overlap as $f=k \delta$, with a stiffness $k$, if $\delta>0$. In tangential direction, the force $f^{t}=k_{t} \delta^{t}$ is proportional to the tangential displacement of the contact points (due to both rotations and sliding) with a stiffness $k_{t}$. The tangential force is limited by Coulombs law for sliding $f^{t} \leq \mu f$, i.e., for $\mu=0$ one has no tangential forces at all. In order to account for energy dissipation the normal and tangential degrees of freedom are also subject to a viscous, velocity dependent damping force. For more details see (Luding 1998; Luding 2006a; Luding 2008).

With tools from numerical integration, as nicely described in textbooks as (Allen and Tildesley 1987; Rapaport 1995), the numerical integration of the equations of motion is then a straightforward exercise.

\section{Ring shear cell simulation in $3 \mathrm{D}$}

The simulation results model a ring-shear cell experiment, as recently proposed (Fenistein and van Hecke 2003; Fenistein, van de Meent, and van Hecke 2004) and simulated (Luding 2004c; Luding 2006b; Ries, Wolf, and Unger 2007). The interesting observation is a universal wide shear zone, initiated at the bottom slit and becoming wider and moving inwards while propagating upwards in the system. 


\section{Model system}

In order to save computing time, only a quarter of the ring-shaped geometry is simulated. The walls are cylindrical, and are rough on the particle scale due to some (about 3 per-cent of the total number) attached particles (Luding 2004c; Luding 2006b). The outer cylinder wall with radius $R_{o}$, and part of the bottom $r>R_{s}$ are rotating around the symmetry axis, while the inner wall with radius $R_{i}$, and the attached bottom-disk $r<R_{s}$ remain at rest. In order to resemble the experiment, the geometry data are $R_{i}=0.0147 \mathrm{~m}$, $R_{s}=0.085 \mathrm{~m}$, and $R_{o}=0.110 \mathrm{~m}$. Note that the small $R_{i}$ value is artificial, but it does not affect the results for the intermediate filling heights used here. For larger filling height, the shearband does not reach the top and other modes of flow can be observed (Fenistein, van de Meent, and van Hecke 2006)

The slit in the bottom wall at $r=R_{s}$ triggers a shear band. In order to examine the behavior of the shear band as function of the filling height $H$, this system was filled with 6000 to 64000 spherical particles with mean radius $\langle a\rangle=1.0 \mathrm{~mm}$ and radii range $0.5 \mathrm{~mm}<a<1.5 \mathrm{~mm}$, see Refs. (Luding 2004c; Luding 2006b). The particles are forced towards the bottom by gravity and are kept inside the system by the cylindrical walls. The moving slit initiates a shear band that moves in wards and becomes wider with increasing height, see Fig. 1

\section{Material and system parameters}

The simulations run for more than $50 \mathrm{~s}$ with a rotation rate $f_{o}=0.01 \mathrm{~s}^{-1}$ of the outer cylinder, with angular velocity $\Omega_{o}=2 \pi f_{o}$. For the average of the displacement, only larger times are taken into account so that the systems quasi-steady state flow is examined - disregarding the transient behavior at the onset of shear.

Two realizations with the same filling height, i.e., $N \approx 37000$ overall particles, are displayed in Fig. 1, both as top- and front-view without $(\mu=0)$ and with friction $(\mu=0.4)$. The color code indicates the displacement and shows that with friction the shear band is narrower than without.

\section{Averaging and micro-macro procedure}

Since we assume translational invariance in the tangential $\phi$-direction, averaging is performed over toroidal volumina and over many snapshots in time (typically 40-60), leading to fields $Q(r, z)$ as function of the radial and 

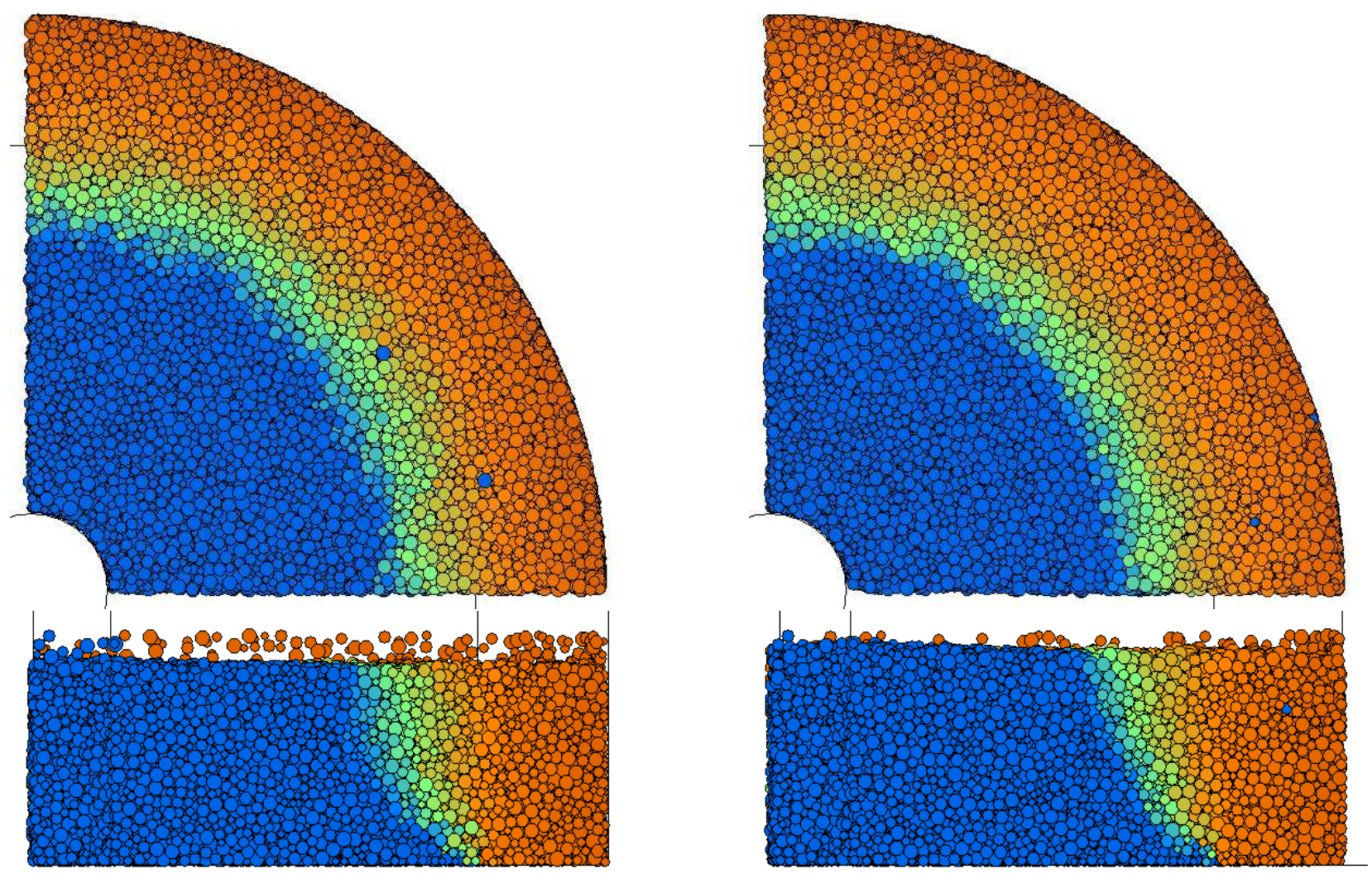

Fig. 1. Snapshots from simulations with different friction coefficients, but the same number of particles, seen from the top and from the front. The mobile particle number is $N=34518$, both without friction $\mu=0$ (Left) and with friction $\mu=0.4$ (Right). The colors blue, green, orange and red denote particles with displacements in tangential direction per second $r \mathrm{~d} \phi \leq 0.5 \mathrm{~mm}, r \mathrm{~d} \phi \leq 2 \mathrm{~mm}$, $r \mathrm{~d} \phi \leq 4 \mathrm{~mm}$, and $r \mathrm{~d} \phi>4 \mathrm{~mm}$, Even though the particle numbers are identical, the filling heights are slightly different: $H \approx 0.037 \mathrm{~m}$ (Left) and $H \approx 0.039 \mathrm{~m}$ (Right).

vertical positions. The averaging procedure was detailed for $2 \mathrm{D}$ systems e.g. in Ref. (Lätzel, Luding, and Herrmann 2000) and will be discussed elsewhere (S. Luding, in preparation) in more detail. The averaging is performed with spacings of $\Delta z \approx 0.0025$ and $\Delta z \approx 0.0028$ in radial and vertical direction, respectively, involving between 30 and 100 particles per averaging cell (toroid), dependent on the radial distance from the center.

Note that scalar quantities like density, coordination number and energy can be averaged straightforwardly - but also vectorial quantities like the velocities or spins of the particles and even tensorial quantities like stress and velocity-gradient can computed. 
Density, coordination number and fabric

From the simulations, one observes that the density, the coordination number and the isotropic fabric decrease with height and are systematically lower in the shear band due to dilatancy.

From two-dimensional simulations, the relation

$$
\operatorname{tr} F=g_{2} \nu C
$$

between density $\nu=\sum_{p \in V} V^{p} / V$, coordination number $C$ and isotropic fabric $\operatorname{tr} F$ was evidenced. The factor $g_{2}$ was found to be a function of the first three moments of the size distribution, and there seems to be no negative effect of friction on the scaling relation, as also observed in 2D (Madadi, Tsoungui, Lätzel, and Luding 2004; Madadi, Peyghoon, and Luding 2005), see Fig. 2.
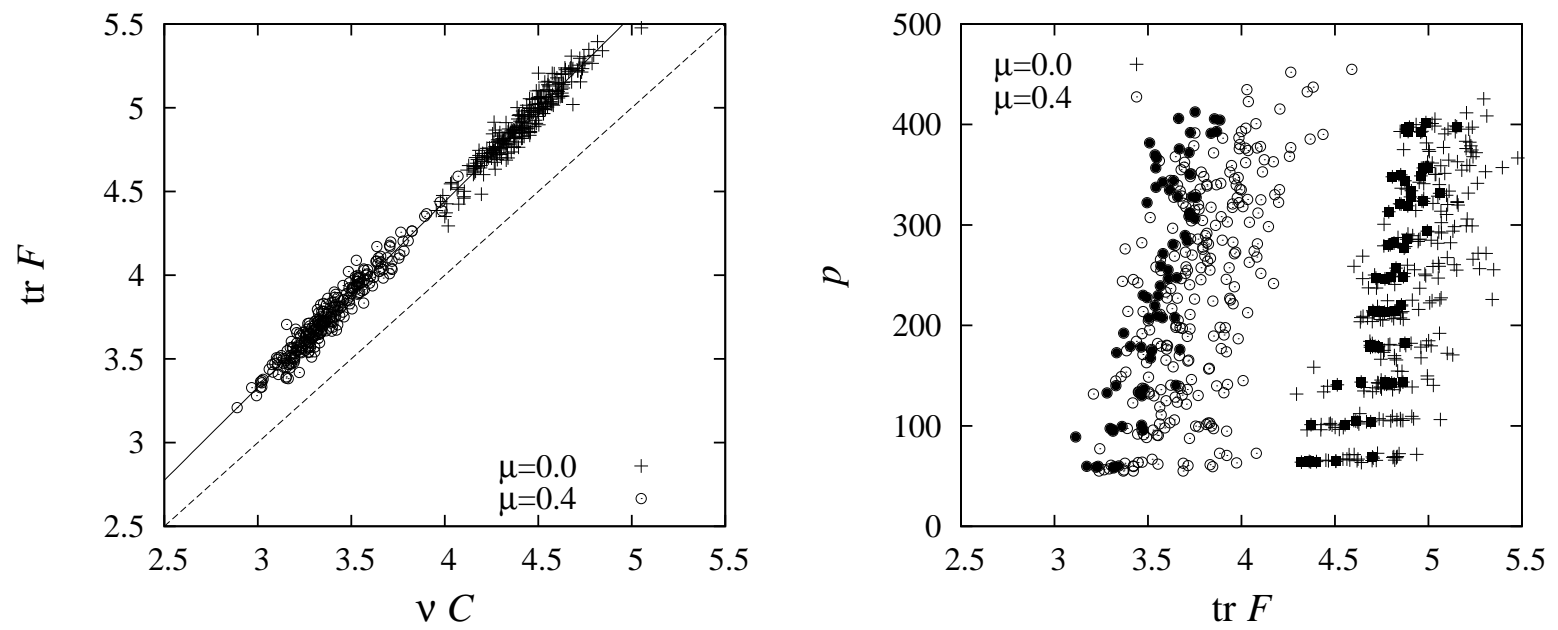

Fig. 2. (Left) Trace of the fabric tensor plotted against the contact number density $\nu C$. The dashed line indicates identity while the solid line is multiplied with a factor $g_{2}=1.1$. Simulation data are taken from the window $0.03 \mathrm{~m} \leq r \leq 0.10 \mathrm{~m}$ and $0.005 \mathrm{~m} \leq z \leq 0.030 \mathrm{~m}$, and we note that data with friction (circles) and without (crosses) collapse on the same line. (Right) Pressure $p$ plotted against $\operatorname{tr} F$ for the same data as in the left panel. The solid symbols are data points for which the strain rate $\dot{\gamma}>0.08 \mathrm{~s}^{-1}$.

Also in the bulk of the present simulation volume (data at the walls and the free surface are not included), the scaling of the isotropic, scalar quantities $g_{2}, \nu, C$, and $\operatorname{tr} F$ works and is not affected by the presence of friction.

Density $\nu$, coordination number $C$ and $\operatorname{tr} F$ are all considerably smaller when friction is active. Without friction, density and coordination numbers are found in the ranges $0.64 \leq \nu \leq 0.68$ and $6.3 \leq C \leq 7.1$, while with friction the ranges are $0.58 \leq \nu \leq 0.66$ and $5.2 \leq C \leq 6.0$. Furthermore, we note that also the isotropic bulk modulus is linearly proportional to the trace of the fabric as it was observed in 2D simulations already. The pressure increases with depth 
inside the material and with $\operatorname{tr} F$. The higher the strain rate the smaller is the contact number density, indicating the effect of dilatancy.

\section{Shear deformation results}

From a set of simulations with different filling heights (data not shown, see (Luding 2004c)), just examined from the top (like in the original experiments), it becomes clear that the shearband moves inwards with increasing filling height and also becomes wider. From the front-view, the same information can be evidenced, see Fig. 1. However the shape of the shear band inside the bulk does not coincide with the shearband on the free-surface: The inwards shift of the shear band takes place deep in the bulk - closer to the surface its position does not change much more.

Comparing the cases with (Right) and without (Left) friction in Fig. 1, the shearband appears more narrow in the presence of friction. In order to allow for a more quantitative analysis of the shear band, both on the top and as function of depth, we perform fits with the universal shape function proposed in Ref. (Fenistein and van Hecke 2003):

$$
v_{\varphi}(r) /\left(r \Omega_{o}\right)=A+B \operatorname{erf}\left(\frac{r-R_{c}}{W}\right)
$$

where $A$ and $B$ are dimensionless amplitudes $A \approx B \approx 0.51 \pm 0.01$, (somewhat smaller for the frictional case), $R_{c}$ is the center of the shearband, and $W$ its width. Note that $R_{c}$ corresponds thus to the maximum of the velocity gradient.

The fits to the simulations confirm the experimental findings in so far that the center of the shear band, as observed on top of the material, moves inwards with a $R_{c} \propto H^{5 / 2}$ behavior, see Refs. (Luding 2004c; Unger, Török, Kertész, and Wolf 2004; Luding 2006b; Cheng, Lechman, Fernandez-Barbero, Grest, Jaeger, Karczmar, Möbius, and Nagel 2006; Török, Unger, Kertesz, and Wolf 2007), and that the width of the shear band increases almost linearly with $H$. Quantitatively speaking, the agreement is $80 \%$, i.e., the simulations without friction lead to a somewhat slower shift of the shearband inwards. For filling heights larger than $H \approx 0.05 \mathrm{~m}$, deviations from this behavior are observed, because the inner cylinder is reached and thus sensed by the shearband. Slower shearing than with $f_{o}=0.01 \mathrm{~s}^{-1}$ does not affect the center position, but reduces slightly the width (data not shown).

The packing is considerably less dense in the presence of friction, which leads to a larger net filling height given the same number of particles. Fur- 
thermore, the filling height varies in radial direction: it is smaller where the velocity gradient is largest. A quantitative study of the velocity gradient leads to the strain rate

$$
\dot{\gamma}=\sqrt{d_{1}^{2}+d_{2}^{2}}=\frac{1}{2} \sqrt{\left(\frac{\partial v_{\phi}}{\partial r}-\frac{v_{\phi}}{r}\right)^{2}+\left(\frac{\partial v_{\phi}}{\partial z}\right)^{2}},
$$

i.e., the shear intensity in the shear plane, as discussed in Ref. (Depken, van Saarloos, and van Hecke 2006), see Eq. (7) therein - note that the solid-body rotation term $v_{\phi} / r$ comes from the cylindrical coordinate system used. The shear planes are in fact described by a normal unit vector $\hat{\gamma}=(\cos \theta, 0, \sin \theta)$, with $\theta=\theta(r, z)=\arccos \left(d_{1} / \dot{\gamma}\right)$, as predicted (Depken, van Saarloos, and van Hecke 2006). The center of the shear band indicates the direction of the unitvector $\hat{\gamma}$. In the system with friction, we observe that the average particles spin is also normal to the shear-plane, i.e., parallel to $\hat{\gamma}$, within the rather strong fluctuations (data not shown).
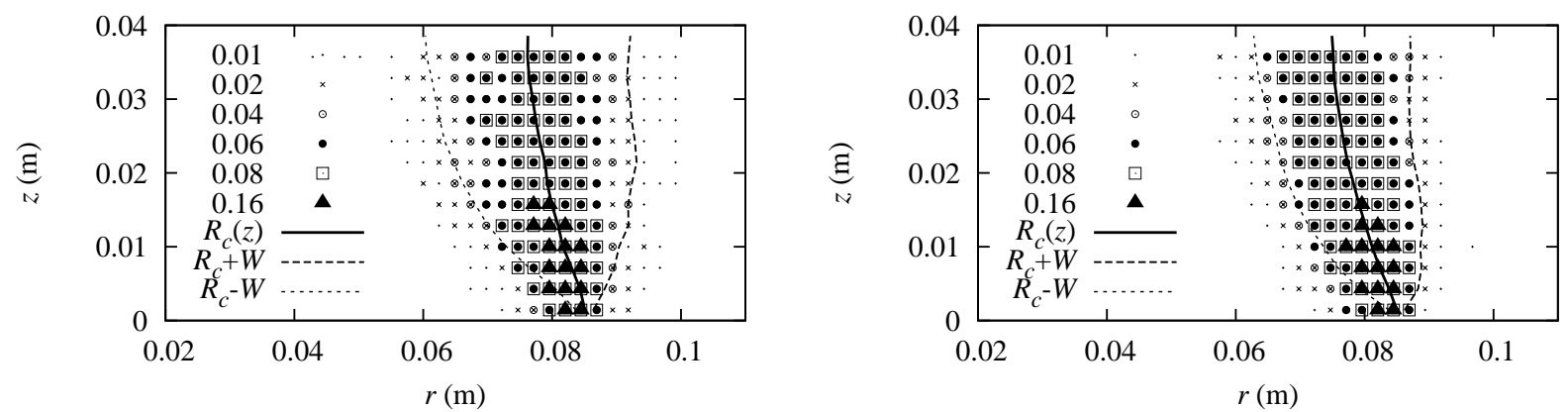

Fig. 3. Graphic representation of the strain rate $\dot{\gamma}$ as function of radial and vertical position for the frictionless (Left) and the frictional (Right) case. Different symbols indicate the magnitude of the strain rate, i.e., $\dot{\gamma} \geq \dot{\gamma}_{i}$, with $\dot{\gamma}_{i}$ given in the inset in units of $\mathrm{s}^{-1}$ - larger symbols correspond also to larger $\dot{\gamma}$. The lines indicate the center $R_{c}$ and the width $W$ of the shear bands, as obtained from the fit function Eq. (2).

The center of the shear band behaves as observed from experiments (Fenistein, van de Meent, and van Hecke 2004; Cheng, Lechman, Fernandez-Barbero, Grest, Jaeger, Karczmar, Möbius, and Nagel 2006) and predicted by theory (Unger, Török, Kertész, and Wolf 2004; Török, Unger, Kertesz, and Wolf 2007), see Fig. 3, for small and moderate filling heights. The presence of friction does not affect the qualitative behavior but the center position and the width: The shear band with friction moves inwards slightly more rapidly and is also somewhat narrower.

This example of a peculiar ring shear cell simulation in 3D shows, that even without complex contact interaction laws, some experiments can be reproduced quantitatively with $80 \%$ agreement. The simulation with friction leads to even 
better agreement of about $90 \%$, but further studies are needed here to establish this trend.

From these simulations, one can also determine the components of the stress tensor

$$
\sigma_{\alpha \beta}=\frac{1}{V} \sum_{c \in V} f_{\alpha} l_{\beta}
$$

with the components of the contact normal forces $f_{\alpha}$ and branch vectors $l_{\beta}$. The sum extends over all contacts within or close to the averaging volume, weighted according to their vicinity - a more detailed description of the averaging procedure and the weighting will be published elsewhere (S. Luding, in preparation). Note that we disregard the tangential forces here for the sake of simplicity. Even though our data (not shown) indicate that the (symmetric) stress is not co-linear with the (symmetrized) strain tensor, we define the shear stress in analogy to the the shear strain as proposed in (Depken, van Saarloos, and van Hecke 2006):

$$
|\tau|=\sqrt{\sigma_{r \phi}^{2}+\sigma_{z \phi}^{2}} .
$$

A more detailed study of the stress- and strain eigensystems indicates ( $\mathrm{S}$. Luding, in preparation) that the eigen-system of the stress is tilted by a few degrees from the eigen-system of the strain, see also Fig. 13 in Ref. (Lätzel, Luding, and Herrmann 2000) for equivalent 2D findings. The co-linearity is stronger for larger shear-rate, however, involving also strong fluctuations of the data (not shown). The non-perfect co-linearity leads to a small systematic error in the shear stress computation.

Remarkably, the shear stress intensity $|\tau| / p \approx \mu$ is almost constant for practically all averaging volumina with strain rates larger than some threshold value, i.e., $\dot{\gamma}>\dot{\gamma}_{c}$, with $\dot{\gamma}_{c} \approx 0.02 \mathrm{~s}^{-1}$. Whether the threshold has a physical meaning or is only an artefact due to the statistical fluctuations in the average data has to be examined further by much longer runs with better statistics.

From the constant shear stress intensity in the shear zone, one can determine the Mohr-Coulomb-type friction angle of the equivalent macroscopic constitutive law, see Fig. 4 , as $\psi \approx \arcsin \mu$. Interestingly, without friction $\psi$ is rather large, i.e., much larger than expected from a frictionless material, whereas it is astonishingly small with friction, i.e., smaller than the microscopic contact friction coefficient $\mu=0.4$ implies.

Plotting the shear stress intensity $|\tau| / p$ in Fig. 5 shows that there is a weak systematic variation of $|\tau| / p$ with pressure: an increase up to $p=200 \mathrm{Nm}^{-2}$ 

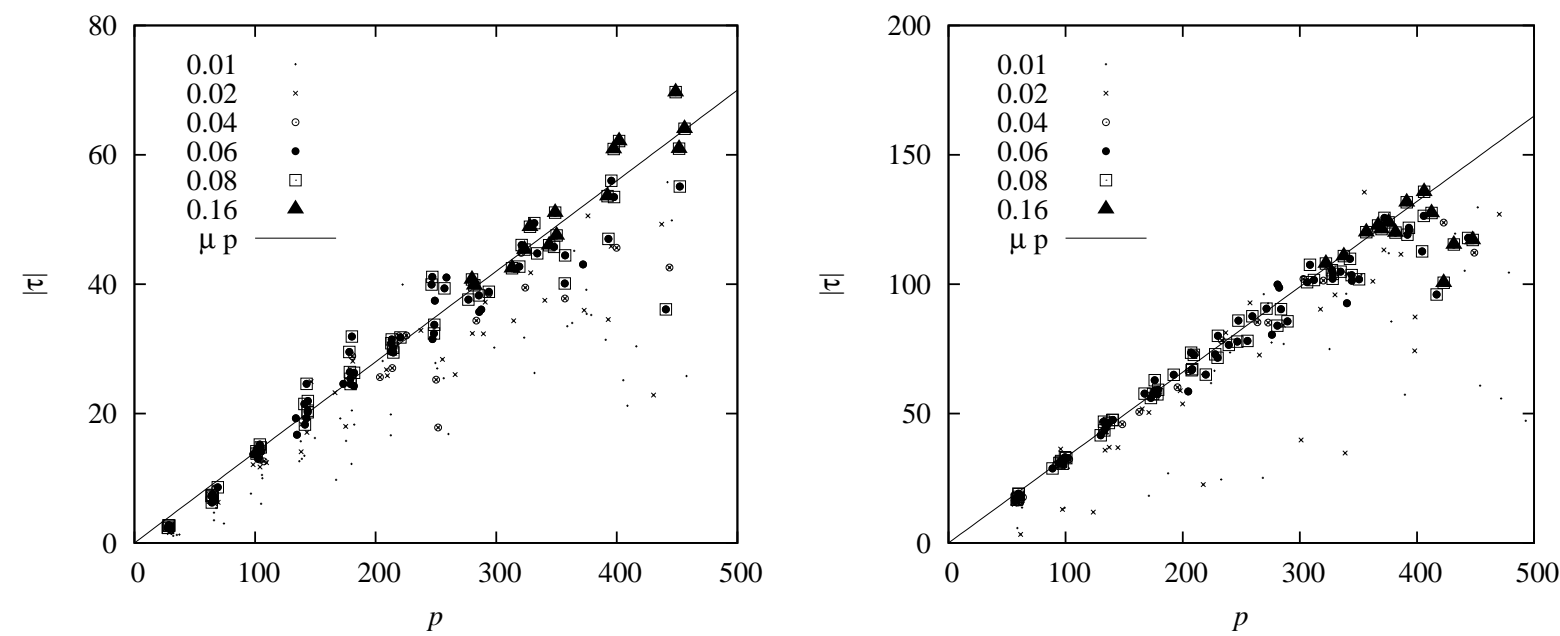

Fig. 4. Shear stress $|\tau|$ plotted against pressure $p$ from the same simulations as in Fig. 3, i.e., the different symbols correspond to different strain rates within the shear planes, as detailed above. The solid line represents the function $\tau_{\max }=\mu p$, with $\mu=0.14$ (Left) and $\mu=0.33$ (Right). Note the different vertical axis scaling of the two plots.
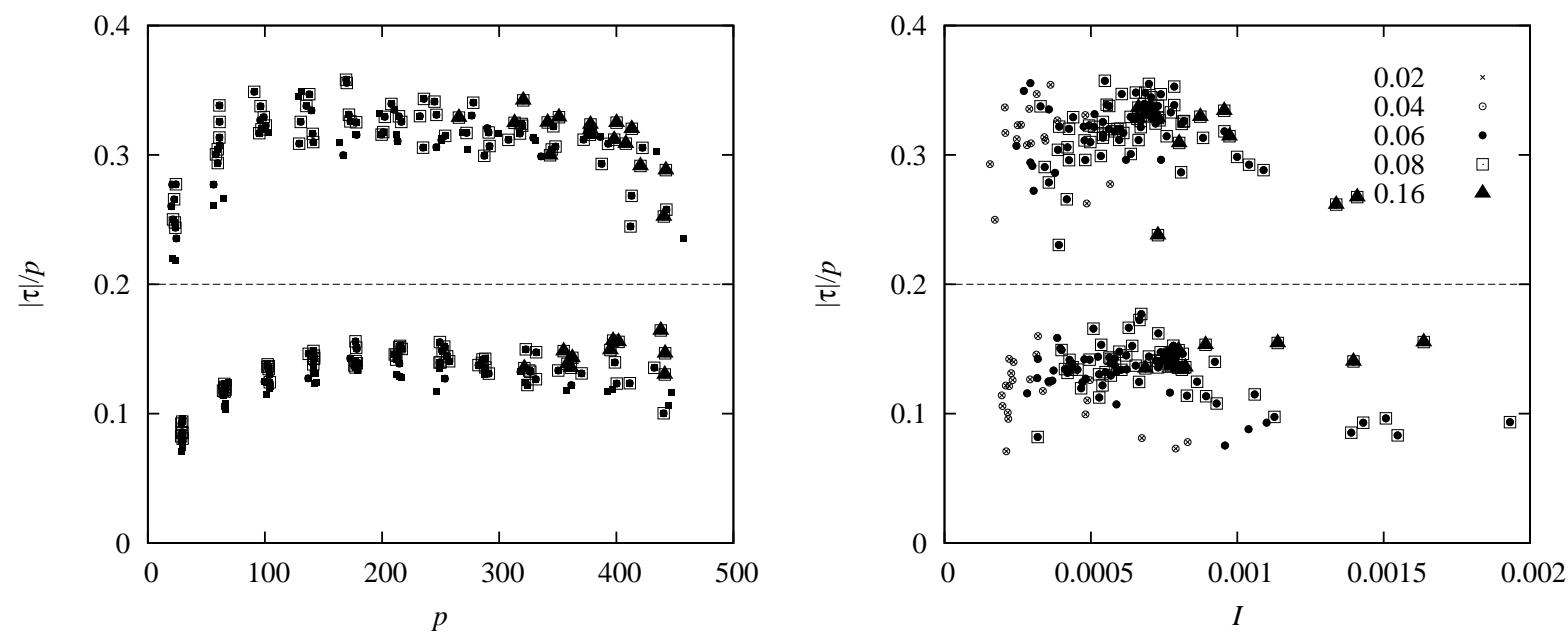

Fig. 5. Shear stress intensity $|\tau| / p$ plotted against pressure (Left) and non-dimensional strain rate $I$ (Right) from the same simulations as in Fig. 3. The dashed line separates the data from simulations without (Bottom) and with (Top) friction.

and a weak decrease for higher $p$. No clear variation of the shear stress intensity with the strain rate $\dot{\gamma}$ can be evidenced, when the latter is non-dimensionalized with the mean diameter $d_{0}$, the pressure $p$ and the density $\varrho$ as proposed in Ref. (GDR MiDi 2004), such that

$$
I=\dot{\gamma} d_{0} \sqrt{\varrho / p} .
$$

The shear stress divided by the shear strain gives something that would be the viscosity in a Newtonian Fluid. The present simulation data show that the "viscosity" decays with $I$, see Fig. 6. Two trends can be seen, namely: (i) the higher the strain rate, the smaller the "viscosity", i.e., $|\tau| / \dot{\gamma} \propto I^{-\alpha}$, 

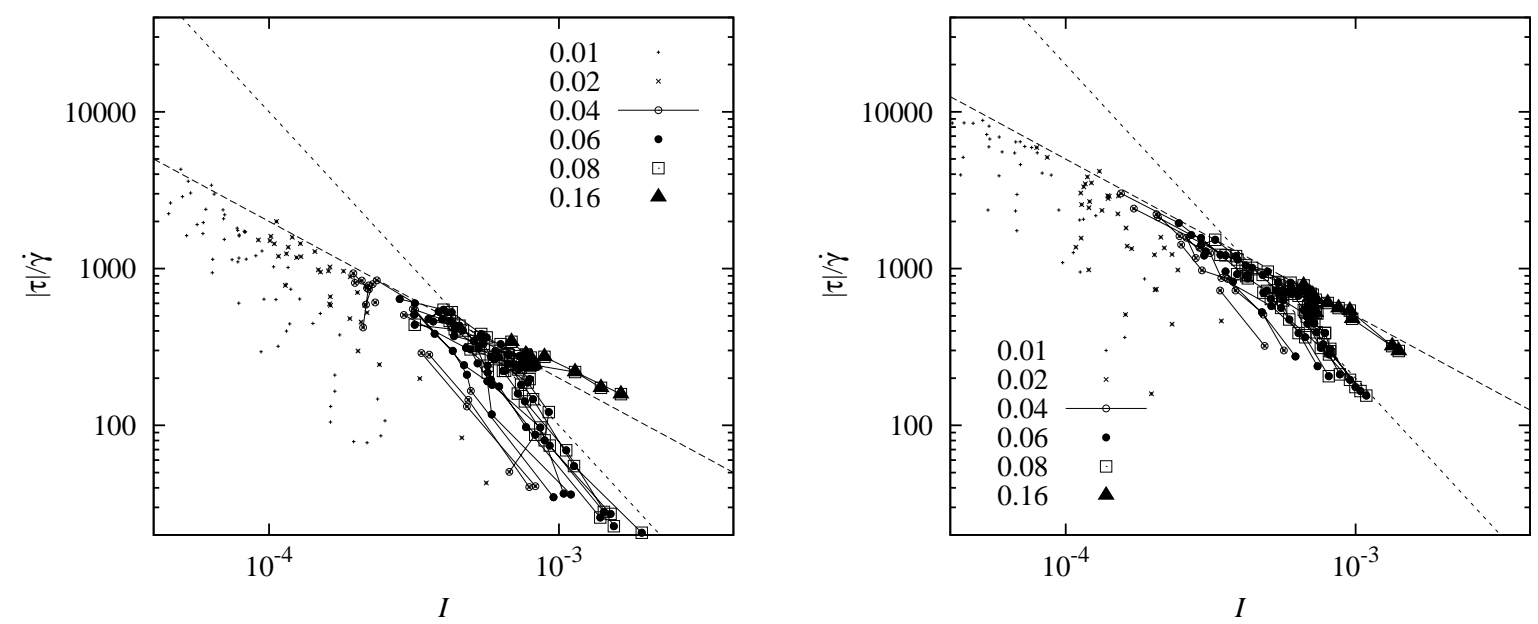

Fig. 6. Shear "viscosity" $|\tau| / \dot{\gamma}$ plotted against the non-dimensional strain rate $I$, for the simulation results without (Left) and with (Right) friction, from the same simulations as in Fig. 3. The dashed and dotted lines indicate slopes -1 and -2 , respectively, while the solid lines connect data points that are located vertically above each other.

with $\alpha \approx 1$, and (ii) the lower the pressure, the smaller the "viscosity", i.e., $|\tau| / \dot{\gamma} \propto p$.

\section{Conclusion}

Two simulations of a slit-bottom Couette ring shear cell show perfect qualitative and good quantitative agreement with experiments. The effect of friction on the results was examined in detail in this study. The frictionless simulations are already in $80 \%$ percent agreement with the experiments - and the simulation with friction comes even close to $90 \%$. This is remarkable, since besides the geometry of the shear cell no special attention was payed to the choice of material parameters, particle-size and particle size distribution.

The isotropic part of the structure (fabric) tensor is proportional to the density, the coordination number, and a dimensionless constant that only depends on the moments of the particle size-distribution function (like in 2D, see (Madadi, Tsoungui, Lätzel, and Luding 2004; Madadi, Peyghoon, and Luding 2005)) - notably this scaling is not at all affected by the presence of friction, even though all the quantities (density, coordination number and fabric-trace) are considerably smaller in the presence of friction. The stress is correlated with the contact number density, $\operatorname{tr} F$, i.e., higher stress leads to larger contact number densities.

From the simulations, we learn that the shear-planes are tilted from the horizontal as proposed in Ref. (Depken, van Saarloos, and van Hecke 2006). The tilt angle can also be seen from the average particle spin (in the presence 
of friction). The shear stress is not exactly co-linear with these shear planes, however, we compute the shear stress as if it would be for the sake of simplicity. The ratio of shear and normal stresses $|\tau| / p$ varies slightly with the pressure, and less clearly with the dimensionless strain rate $I$. Astonishingly, the shear stress intensity, $|\tau| / p$, is almost constant within the wide shear band, corresponding to a Mohr-Coulomb type yield surface with effective friction angles of $\psi \approx 8^{\circ}$ and $\psi \approx 19^{\circ}$ for the microscopic friction coefficients $\mu=0$ and $\mu=0.4$, respectively.

The "viscosity", i.e., the ratio of shear stress and strain rate, $|\tau| / \dot{\gamma}$, decays as $I^{-1}$ and is proportional to $p$, indicating that neither $p$ nor $I$ are the only control parameters for this geometry. A more detailed study of the anisotropy of the stress and the structure tensors, and their relative orientations to the shear plane should unravel the constitutive relations needed to describe the wide shear bands in the framework of a continuum theory.

\section{Acknowledgements}

We acknowledge the financial support of several funding institutions that supported the reviewed research, the Deutsche Forschungsgemeinschaft (DFG), and the Stichting voor Fundamenteel Onderzoek der Materie (FOM), financially supported by the Nederlandse Organisatie voor Wetenschappelijk Onderzoek (NWO). Furthermore, helpful discussions with Martin van Hecke, Dietrich Wolf and Niels Kruyt are acknowledged.

\section{References}

Allen, M. P. and D. J. Tildesley (1987). Computer Simulation of Liquids. Oxford: Oxford University Press.

Cheng, X., J. B. Lechman, A. Fernandez-Barbero, G. S. Grest, H. M. Jaeger, G. S. Karczmar, M. E. Möbius, and S. R. Nagel (2006). Three-dimensional shear in granular flow. Phys. Rev. Lett. 96, 038001.

Depken, M., W. van Saarloos, and M. van Hecke (2006). Continuum approach to wide shear zones in quasistatic granular matter. Phys. Rev. E 73, 031302.

Fenistein, D., J. W. van de Meent, and M. van Hecke (2004). Universal and wide shear zones in granular bulk flow. Phys. Rev. Lett. 92, 094301. e-print cond-mat/0310409.

Fenistein, D., J.-W. van de Meent, and M. van Hecke (2006). Core precession 
and global modes in granular bulk flow. Phys. Rev. Lett. 96, 118001.

Fenistein, D. and M. van Hecke (2003). Kinematics - wide shear zones in granular bulk flow. Nature 425(6955), 256.

GDR MiDi (2004). On dense granular flows. Eur. Phys. J. E 14, 341-365. Herrmann, H. J., J.-P. Hovi, and S. Luding (Eds.) (1998). Physics of dry granular media - NATO ASI Series E 350, Dordrecht. Kluwer Academic Publishers.

Kishino, Y. (Ed.) (2001). Powders \& Grains 2001, Rotterdam. Balkema. Lätzel, M., S. Luding, and H. J. Herrmann (2000). Macroscopic material properties from quasi-static, microscopic simulations of a two-dimensional shear-cell. Granular Matter 2(3), 123-135. e-print cond-mat/0003180.

Lätzel, M., S. Luding, and H. J. Herrmann (2001). From discontinuous models towards a continuum description. In P. A. Vermeer, S. Diebels, W. Ehlers, H. J. Herrmann, S. Luding, and E. Ramm (Eds.), Continuous and Discontinuous Modelling of Cohesive Frictional Materials, Berlin, pp. 215-230. Springer.

Lätzel, M., S. Luding, H. J. Herrmann, D. W. Howell, and R. P. Behringer (2003). Comparing simulation and experiment of a $2 \mathrm{~d}$ granular couette shear device. Eur. Phys. J. E 11(4), 325-333.

Luding, S. (1998). Collisions \& contacts between two particles. In H. J. Herrmann, J.-P. Hovi, and S. Luding (Eds.), Physics of dry granular media - NATO ASI Series E350, Dordrecht, pp. 285. Kluwer Academic Publishers.

Luding, S. (2004a). Micro-macro models for anisotropic granular media. In P. A. Vermeer, W. Ehlers, H. J. Herrmann, and E. Ramm (Eds.), Modelling of Cohesive-Frictional Materials, Leiden, Netherlands, pp. 195-206. Balkema. (ISBN 041536023 4).

Luding, S. (2004b). Micro-macro transition for anisotropic, frictional granular packings. Int. J. Sol. Struct. 41, 5821-5836.

Luding, S. (2004c). Molecular dynamics simulations of granular materials. In H. Hinrichsen and D. E. Wolf (Eds.), The Physics of Granular Media, Weinheim, Germany, pp. 299-324. Wiley VCH.

Luding, S. (2005a). Anisotropy in cohesive, frictional granular media. $J$. Phys.: Condens. Matter 17, S2623-S2640.

Luding, S. (2005b). Shear flow modeling of cohesive and frictional fine powder. Powder Technology 158, 45-50.

Luding, S. (2006a). About contact force-laws for cohesive frictional materials in $2 \mathrm{~d}$ and $3 \mathrm{~d}$. In P. Walzel, S. Linz, C. Krülle, and R. Grochowski 
(Eds.), Behavior of Granular Media, pp. 137-147. Shaker Verlag. Band 9, Schriftenreihe Mechanische Verfahrenstechnik, ISBN 3-8322-5524-9.

Luding, S. (2006b). Particulate solids modeling with discrete element methods. In P. Massaci, G. Bonifazi, and S. Serranti (Eds.), CHoPS-05 CD Proceedings, Tel Aviv, pp. 1-10. ORTRA.

Luding, S. (2008). Cohesive frictional powders: Contact models for tension. Granular Matter 10 (3), in press.

Luding, S., M. Lätzel, and H. J. Herrmann (2001). From discrete element simulations towards a continuum description of particulate solids. In A. Levy and H. Kalman (Eds.), Handbook of Conveying and Handling of Particulate Solids, Amsterdam, The Netherlands, pp. 39-44. Elsevier. Madadi, M., S. M. Peyghoon, and S. Luding (2005). Stress and fabric for polydisperse, frictionless, dense $2 \mathrm{~d}$ granular media. In R. Garcia-Rojo, H. J. Herrmann, and S. McNamara (Eds.), Powders and Grains 2005, Leiden, Netherlands, pp. 93-97. Balkema.

Madadi, M., O. Tsoungui, M. Lätzel, and S. Luding (2004). On the fabric tensor of polydisperse granular media in 2d. Int. J. Sol. Struct. 41(9-10), 2563-2580.

Rapaport, D. C. (1995). The Art of Molecular Dynamics Simulation. Cambridge: Cambridge University Press.

Ries, A., D. E. Wolf, and T. Unger (2007). Shear zones in granular media: Three-dimensional contact dynamics simulation Phys. Rev. E 76, 051301. Török, J., T. Unger, J. Kertesz, and D. E. Wolf (2007). Shear zones in granular materials: Optimization in a self-organized random potential. Phys. Rev. E 75, 011305.

Unger, T., J. Török, J. Kertész, and D. Wolf (2004). Shear band formation in granular media as a variational problem. Phys. Rev. Lett. 92, 214301. Vermeer, P. A., S. Diebels, W. Ehlers, H. J. Herrmann, S. Luding, and E. Ramm (Eds.) (2001). Continuous and Discontinuous Modelling of Cohesive Frictional Materials, Berlin. Springer. Lecture Notes in Physics 568. 